\title{
Für eine Ethik der Zukunftsverantwortung
}

\author{
Während Corporate Social Responsibility, Corporate Governance oder Corporate \\ Sustainability in aller Munde sind, ist die Verknüpfung von Generationenge- \\ rechtigkeit und Unternehmenspolitik neu. Sie ist jedoch hochaktuell, denn Trend- \\ forscher prognostizieren, dass Generationengerechtigkeit in den nächsten \\ Jahren das Schlïsselwort unserer Gesellschaft wird.
}

$\mathrm{D}$ er Begriff ,Generationengerechtigkeit" erobert die politische Agenda. Im Grundsatzprogramm der Grünen ist ihm ein Unterabschnitt gewidmet, auch in der Diskussion um ein neues Grundsatzprogramm der SPD spielt er eine zentrale Rolle. In den Wiesbadener Grundsätzen der FDP wird er bereits häufiger verwandt als ,soziale Gerechtigkeit“" Während es immer noch höchst unterschiedliche Definitionen von Nachhaltigkeit gibt, gibt der Begriff Generationengerechtigkeit von der Wortbedeutung her einen engeren Spielraum vor. Er benennt seine Subjekte, Generationen, und er bindet sich inhaltlich an die Ethik, die den Begriff der Gerechtigkeit vergleichsweise klar umrissen hat. Ein weiterer Vorteil der Begriffe Generationengerechtigkeit beziehungsweise generationengerechte Unternehmenspolitik ist auch, dass beide unmittelbar zum Nachdenken anregen und Energien zum Handeln freisetzen.

Je schwammiger der Begriff Nachhaltigkeit wird, desto mehr wächst der Bedarf nach einem neuen Leitbild für unternehmerisches und gesamtgesellschaftliches Handeln. Gerechtigkeit zwischen Alt und Jung sowie zwischen heutigen und zukünftigen Generationen ist eine der wichtigsten Begründungen, warum wir Umwelt und Natur schützen sollten (1). Er umfasst ein komplettes politisches Programm - von der Umwelt-, über die Finanz- bis hin zur Bildungspolitik. Wie aber lässt sich der Begriff wirklich definieren - und wie lässt er sich in Bezug setzen zu unternehmerischem Handeln?

\section{Definition von Generationen- gerechtigkeit}

Generationengerechtigkeit setzt sich aus den Einzelwörtern Generationen und Gerechtigkeit zu- sammen. Laut Herkunftsduden ist eine Generation die „Gesamtheit aller etwa zur gleichen Zeit geborenen Menschen“. Der Substantiv wurde im 17. Jhdt. aus lateinisch generatio (Zeugungsfähigkeit) entlehnt. Heute bezeichnet der Begriff Generation erstens die Altersspanne, bis aus Kindern Eltern und aus Eltern Großeltern werden. In Deutschland gebären Frauen heute durchschnittlich das erste Kind mit etwa 29 Jahren. Man spricht daraus abgeleitet bei den etwa Unterdreißigjährigen von der jungen, bei den 30-60jährigen von der mittleren und bei den Übersechzigjährigen von der älteren Generation.

Zweitens wird das Wort Generation in der englischen, der deutschen und zahlreichen weiteren Sprachen verwandt, um die Gesamtheit der heute lebenden Menschen zu bezeichnen. In diesem Sinn lebt jeweils nur eine Generation zur gleichen Zeit. Was bedeutet dies etwa für die Aussage eines 28jährigen: „Meine Generation wird benachteiligt, aber die nach uns kommende Generation wird noch mehr benachteiligt sein"? In der erstgenannten, engen Definition wäre der sprachliche Ausdruck ,,meine Generation“, bezogen auf Deutschland, gleichbedeutet mit 26,7 Millionen Menschen. Die Designate dieser Definition wären die Altersgruppen 0 bis 30 Jahre. Verwendete er dagegen bei seiner sprachlichen Äußerung die weite Definition, so würden 82,4 Millionen Menschen darunter fallen.

Die klare Unterscheidung zwischen beiden Bedeutungen wurde in der wissenschaftlichen Debatte über Generationengerechtigkeit lange vernachlässigt. So kreiste die Debatte lange ausschließlich um die weite Definition, wobei das Wohl zukünftiger Generationen zur Debatte stand. Es wurde diskutiert, wie man eine Interessensvertretung für heute noch nicht Geborene einrichtet. Eine erhebliche Veränderung der Debatte ergibt sich, wenn man zusätzlich die enge Definition zu Grunde legt und dann von nachrückenden Generationen spricht. Die Bezeichnung nachrückende Generationen umfasst im Gegensatz zum Begriff zukünftige Generationen nicht nur die noch nicht geborenen Generationen, sondern darüber hinaus auch bereits lebende Kinder und Jugendliche. Folgende Terminologie bringt dies zum Ausdruck: Wird Generation im Sinne der engen Bedeutung gebraucht, so sprechen wir von temporaler Generationengerechtigkeit, sonst von intertemporaler Generationengerechtigkeit. Temporale Generationengerechtigkeit ist also die Gerechtigkeit zwischen jungen, mittelalten und älteren lebenden Menschen. Intertemporale Generationengerechtigkeit wird definiert als die Gerechtigkeit zwischen Menschen, die früher lebten, die heute leben und die zukünftig leben werden.

Das Wort Generationengerechtigkeit als Zusammensetzung der Worte Generationen im Plural und Gerechtigkeit kann gemäß seinem inneren Wortsinn nur Gerechtigkeit zwischen den Generationen, nicht innerbalb einer Generation bedeuten. Geschlechtergerechtigkeit - um ein verwandtes Wort zu nehmen - bedeutet auch Gerechtigkeit zwischen Männern und Frauen und nicht innerhalb der Gesamtheit der Frauen. Versuche, Generationengerechtigkeit als Überbegriff sowohl für intra- als auch für intergenerationelle Gerechtigkeit umzudefinieren, sind demnach nicht zulässig, da eine solch weite Definition Erkenntnismöglichkeiten abschneiden und zumal gegen grammatikalische Regeln verstoßen würde.

\section{Gerechtigkeit als Gleichheit}

Eine erste Arbeitsdefinition von Generationengerechtigkeit geht von dem Grundsatz aus, dass es ungerecht sei, Gleichartiges oder Gleichwertiges ungleich zu behandeln. Artikel 3 Absatz 1 des deutschen Grundgesetzes bestimmt: „Alle Menschen sind vor dem Gesetz gleich." Das Bundesverfassungsgericht entnimmt der Vorschrift das Gebot, Gleiches gleich, Ungleiches seiner Eigenart entsprechend verschieden zu behandeln. Dieser Ansatz ist konsistent mit dem Gebrauch des Gerechtigkeitsbegriffs in anderen Zusammenhängen: Bei Geschlechtergerechtigkeit wird die Ungleichbehandlung aufgrund des Geschlechts gebrandmarkt, sofern nicht objektive Gründe sie erfordern. Ebenso empfindet man es weltweit als ungerecht, wenn Menschen nur aufgrund ihrer Hautfarbe unterschiedlich behandelt werden. Betrachtet man Generationen als gleich- 
wertig und folgt dem Gleichbehandlungsgrundsatz, so könnte die erste Arbeitsdefinition von Generationengerechtigkeit lauten, dass keine Generation besser oder schlechter gestellt werden sollte als eine andere.

Generationengerechtigkeit kann jedoch auch weitergehend definiert werden. Nicht nur eine Nicht-Schlechterstellung, sondern eine Besserstellung kommender Generationen sei ethisch geboten und deshalb anzustreben. Die bisherigen Generationen haben Wachstum und Wohlstandszunahme erfahren, sie sollten im Sinne der Gerechtigkeit dafür sorgen, dass dies auch für zukünftige Generationen möglich bleibt. Es war das Motiv früherer Eltern, dass es ihren Kindern einmal besser gehen soll. Eine positive intergenerationelle Sparrate sollte somit auch das Ziel der heute Lebenden im Umgang mit zukünftigen Generationen sein. Fortschritt in der Lebensqualität ist sicherlich wünschenswerter als ein Stillstand auf dem Status Quo, letzterer wiederum ist einer Verschlechterung vorzuziehen. Für diese Erweiterung lässt sich auch ins Felde führen, dass es niemals gelingen wird, rechnerisch exakt einen Zustand der Gleichbehandlung herbeizuführen. Um nicht das Risiko einer versehentlichen Schlechterstellung der Zukünftigen einzugehen, sollten die Heutigen im Sinne des Vorsorgeprinzips eine Besserstellung anstreben. Auch nach einer teleologischen Ethik, also vom Ziel her gedacht, ist es plausibel, dass eine Welt, in der nach dem weitergehenden moralischen Postulat gelebt wird, insgesamt wünschenswerter wäre, da der Fortschritt hin zum Besseren dort schneller wäre. Nicht zuletzt ist es nach dem diskursethischem Kriterium ,gerecht ist, was allen Beteiligten eines herrschaftsfreien Diskurses als gerecht erscheint" so, dass die versuchte Besserstellung der Zukünftigen große Zustimmung erfährt. Intertemporale Generationengerechtigkeit kann also folgendermaßen definiert werden:

- Generationengerechtigkeit ist erreicht, wenn die Chancen zukünftiger Generationen auf Befriedigung ihrer eigenen Bedürfnisse mindestens so groß sind wie die der heutigen Generation.

In der Definition für temporale Generationengerechtigkeit muss man ,zukünftige Generationen“ durch ,nachrïckende Generationen“ und ,heutige Generation“ durch ,heute mittlere und ältere Generationen“ ersetzen. Alternativ kann man dann auch schreiben:
- Generationengerechtigkeit ist erreicht, wenn die Chancen nachrückender Generationen auf Befriedigung ihrer eigenen Bedürfnisse mindestens so groß sind wie die der ihnen vorangegangenen Generationen.

\section{Unternehmen als Träger von Generationengerechtigkeit}

Mit der Fokussierung auf die zweite Definition wird das Konzept vor allem für drei unternehmerische Handlungsfelder relevant: Umweltfreundlichkeit der Produktion, Ausbildungsplätze und Betriebsrenten. Alle drei Felder haben einen klaren Zeitbezug, der mehrere hintereinanderfolgende, heute lebende Generationen umfasst.

1.) Ausbildungsplätze für die junge Generation: Mehr als in jeder anderen Lebensphase ist es für einen Menschen nach dem Schulabschluss notwendig, den Schritt ins Erwerbsleben zu schaffen.

2.) Ökologische Unternehmensfiuhrung: Die Entscheidungen von Managern und Unternehmern bestimmen maßgeblich, welches Naturkapital nachrückende Generationen noch nutzen und genießen können.

3.) Die Problematik der Betriebsrenten: In vielen Unternehmen haben nur die älteren Arbeitnehmer noch eine Betriebsrente. Diese Schlechterstellung der jüngeren Generation ist ein eklatanter Verstoß gegen das Prinzip der Generationengerechtigkeit.

Unternehmen haben, solange sie einen angemessenen Gewinn erzielen, Freiheitsgrade zum ethischen Handeln. Aus ihrer Binnensicht stellen sich die Fragen: Ist Corporate Generational Responsibility ein geeignetes Leitbild für das Unternehmen? Wie sähe eine generationengerechte Unternehmenspolitik unter sozialen als auch ökologischen Aspekten in meinem Unternehmen konkret aus? Der Unternehmenserfolg ist aber auch von dem Verhalten externer Akteure und den staatlichen Rahmenbedingungen abhängig. Konsumenten können z.B. generationengerecht handelnde Unternehmen belohnen, indem sie deren Produkte kaufen. Kritische Aktionäre können zum Beispiel als Eigentümer auf der Hauptversammlung Druck auf das Management ausüben, mehr Bewusstsein für die Nachwelt zu zeigen. Nichtregierungsorganisationen können Unternehmen öffentlich loben oder an den Pranger stellen.

Generationengerechtes Handeln ist eine Möglichkeit von ethischem unternehmerischem Handeln, eine Schwerpunktsetzung. Ein Unter- nehmen, welches sich für eine solche Strategie entschieden hat, erzielt zunächst einmal Wirkungen „nach innen“, also auf die eigenen Arbeitnehmer. Höhere Arbeitszufriedenheit, geringere Fluktuation, höhere Motivation und eine entsprechende ,Mitarbeiter-PR' sind Effekte, deren ökonomischer Wert außer Frage steht, aber nicht immer präzise in Zahlen ausgedrückt werden kann. Dies gilt allerdings für jede ethisch motivierte Strategie. Darüber hinaus kann aber die systematische Beschäftigung mit der Zukunft im Rahmen von Corporate Generational Responsibility auch ganz allgemein den Blick für die Zukunft schärfen und helfen, langfristig zu denken.

\section{$\checkmark$ Fazit}

Generationengerechtes Handeln wird bisher vor allem von der Politik eingefordert, zum Beispiel im Hinblick auf die Reform der Sozialversicherungssysteme. Aber auch Unternehmen, gerade wenn sie multinational tätig sind, sind wichtige Akteure in der Gesellschaft und tragen als solche Verantwortung. Im Jahre 1997 fanden sich unter den 100 größten Budgets der Welt nur noch 34 Staatshaushalte, die restlichen 66 Plätze wurden bereits von milliardenstarken transnationalen Konzernen eingenommen. Unternehmen können heutzutage die Chancen der Globalisierung nutzen und ihre Produktion in die Länder mit den niedrigsten Umwelt- und Sozialstandards verlegen. Sie können in der Dritten Welt Kinder unter ausbeuterischen Bedingungen arbeiten lassen und in der ersten Welt Ausbildungsplätze streichen. Sie haben aber auch die Wahl, sich im Rahmen ihrer Möglichkeiten verantwortlich zu verhalten. Eine Unternehmensethik, die Generationengerechtigkeit einbezieht, ist daher dringend erforderlich.

\section{Anmerkung}

(1) Vgl.: Tremmel, Jörg: Generationengerechtigkeit - Versuch einer Definition, in: Stiftung für die Rechte zukünftiger Generationen ( $\mathrm{Hg}$. ): Handbuch Generationengerechtigkeit. München 2003.

\section{Der Autor}

Jörg Tremmel ist geschäftsführender Vorstand der Stiftung für die Rechte zukünftiger Generationen (SRzG).

Kontakt: SRzG, Postfach 5115, 61422 Oberursel, Tel. 06171-982367, Fax 06171-952566, Web: www.srzg.de, E-mail: tremmel@srzg.de 
(c) 20I0 Authors; licensee IÖW and oekom verlag. This is an article distributed under the terms of the Creative Commons Attribution Non-Commercial No Derivates License (http://creativecommons.org/licenses/by-nc-nd/3.o/), which permits unrestricted use, distribution, and reproduction in any medium, provided the original work is properly cited. 